ORIGINAL ARTICLE

\title{
Effective ways of restoring muscular imbalances of the rotator cuff muscle group: a comparative study of various training methods
}

\author{
P C Malliou, K Giannakopoulos, A G Beneka, A Gioftsidou, G Godolias
}

Br J Sports Med 2004;38:766-772. doi: 10.1136/bjsm.2003.009548

See end of article for authors' affiliations

Correspondence to

Dr Beneka, Department of

Physical Education and

Sport Science, Democritus

University of Thrace,

Komotini 69100, Greece;

abeneka@yahoo.gr

Accepted

16 December 2003
Background: Many studies have compared different training methods for improving muscular performance, but more investigations need to be directed to the restoration of muscular imbalances.

Objective: To determine the most effective training for altering strength ratios in the shoulder rotator cuff. Methods: Forty eight physical education students were randomly assigned to four groups (12 per group): (a) experimental group who carried out multijoint dynamic resistance training for shoulder internal and external rotation movement (pull ups or lat pull downs, overhead press, reverse pull ups, push ups) (MJDR group); (b) experimental group who exercised the same muscle group using dumbbells weighing $2 \mathrm{~kg}$ (isolated group); (c) experimental group who followed an isokinetic strengthening programme for the rotator cuff muscle group (isokinetic group); (d) control group who had no strength training. Testing was performed in the supine position with the glenohumeral joint in $90^{\circ}$ of abduction in the coronal plane, with a range of motion of $0-90^{\circ}$ of external rotation and $0-65^{\circ}$ of internal rotation at angular velocities of 60 , 120 , and $180 \%$ s. The test procedure was performed before and after the exercise period of six weeks. Results: One way analysis of variance found no differences between the groups for the initial tests. Analysis of variance with repeated measures showed that the strength ratios in all the experimental groups had altered after the exercise period, with the isokinetic group showing the most significant improvement. Conclusions: Isokinetic strengthening is the most effective method of altering strength ratios of the rotator cuff muscle.
$\mathrm{T}$ raining for sports that involve throwing (baseball, volleyball, tennis, and shotput) requires the athlete to propel an object horizontally and vertically, taking into consideration that the abducted and externally rotated position of the glenohumeral joint during the cocking phase needs dynamic muscular stabilisation to maintain humeral head congruency.

The functional role of the rotator cuff requires objective evaluation to detect possible imbalances. Sahrmann ${ }^{2}$ and Caillet $^{3}$ define muscle imbalance as a failure of the agonistantagonist relation, when agonistic/antagonistic muscle groups function cooperatively to control the joints that they cross. Therefore the term balance refers to the balance between the torque ratio of agonistic and antagonistic muscle groups. ${ }^{4}$ Information on shoulder strength has been obtained by isokinetic testing performed on athletes at the high school, collegiate, and professional levels of throwing ${ }^{6-9}$ and elite level tennis players. ${ }^{10}$ The external rotators of the dominant side are often weak in relation to those of the nondominant side. Testing at $90^{\circ}$ of abduction appears to be the most sensitive way of detecting differences ${ }^{911}$ compared with other shoulder testing positions.

Several studies ${ }^{12}{ }^{13}$ have examined the effectiveness of different types of training, but have investigated only leg muscles. The results cannot be extrapolated to the upper limb because the muscle groups have different training adaptations, ${ }^{14}$ which are due to differences in familiarity with concurrent bilateral activation. Therefore further study is required to examine the effects of strength training on the rotator cuff muscle ratio.

A comparative study of isokinetic exercise training and multijoint dynamic resistance (MJDR) training was conducted by Beneka et $a l^{4}$ in the rotator cuff muscle group. The results show that MJDR training is a simple way to improve rotator cuff muscular performance. It can be almost as effective as isokinetic exercise, but further study is required to investigate ways to restore possible muscular imbalances. Only the improvement in peak torque between the two limbs was studied but not muscle imbalances.

With regard to the type of training that is most effective in strengthening the rotator cuff muscles, Brostrom et $a l^{5}$ recommended "isolated" types of exercise, which better emphasise recruitment of the muscles in question. This means that, after evaluation of muscular performance and detection of possible imbalances in strength, an exercise programme with isolated movements is applied focusing on the weak muscles.

According to previous studies, each type of exercise training (isokinetic, dynamic resistance, and isolated) is effective, but dynamic resistance exercise has only been applied using isotonic devices with no MJDR exercises incorporated. We found no studies that examined all three training modalities. The aim of this study was to compare the three training modes in the same participants and the same muscle groups, to determine the most effective way of altering the strength ratios. This was assessed by measuring isokinetic muscular performance before and after the exercise period. We chose to compare these training protocols because athletic trainers and practitioners often need to strengthen weak muscle groups. We also thought that it would be useful to know which would be the most efficient at restoring muscular imbalances even if they had different characteristics.

\section{MATERIALS AND METHODS Subjects}

Forty eight physical education students volunteered. They had a mean (SD) age of 22.3 (1.2) years, a mean (SD) weight 
Table 1 Strengthening programmes of the experimental groups during the training period

\begin{tabular}{|c|c|c|c|c|c|c|c|}
\hline Group & Exercise & Week 1 & Week 2 & Week 3 & Week 4 & Week 5 & Week 6 \\
\hline \multirow[t]{4}{*}{ Isolated } & IR right & $3(8-15)$ & $3(8-15)$ & $4(8-15)$ & $5(8-15)$ & $6(8-15)$ & $7(8-15)$ \\
\hline & IR left & $3(8-15)$ & $3(8-15)$ & $4(8-15)$ & $5(8-15)$ & $6(8-15)$ & $7(8-15)$ \\
\hline & ER right & $3(8-15)$ & $3(8-15)$ & $4(8-15)$ & $5(8-15)$ & $6(8-15)$ & $7(8-15)$ \\
\hline & ER left & $3(8-15)$ & $3(8-15)$ & $4(8-15)$ & $5(8-15)$ & $6(8-15)$ & 7 (8-15) \\
\hline \multirow[t]{4}{*}{ MJDR } & Pull up & $3(8-15)$ & $3(8-15)$ & $4(8-15)$ & $5(8-15)$ & $6(8-15)$ & $7(8-15)$ \\
\hline & Overhead press & $3(8-15)$ & $3(8-15)$ & $4(8-15)$ & $5(8-15)$ & $6(8-15)$ & $7(8-15)$ \\
\hline & Reverse pull up & $3(8-15)$ & $3(8-15)$ & $4(8-15)$ & $5(8-15)$ & $6(8-15)$ & $7(8-15)$ \\
\hline & Push ups & $3(8-15)$ & $3(8-15)$ & $4(8-15)$ & $5(8-15)$ & $6(8-15)$ & $7(8-15)$ \\
\hline Isokinetic & Internal/external movement & $10(10)$ & $10(10)$ & $10(10)$ & $10(10)$ & $10(10)$ & $10(10)$ \\
\hline
\end{tabular}

The values are number of sets with the number of repetitions in parentheses.

$\mathrm{IR}$, Internal rotation; ER, external rotation; MJDR, multijoint dynamic resistance.

of $72.3(3.8) \mathrm{kg}$, and a mean (SD) height of $178.2(3.2) \mathrm{cm}$. They had been free from shoulder injury over the preceding two years, had full range of motion in the testing position, and they performed no additional physical activity during the research period. Procedures were in accordance with the ethical standards of the committee on human experimentation at the institution at which the work was conducted and with the Helsinki declaration of 1975.

\section{Instrumentation}

All isokinetic testing was performed with a Cybex 6000 isokinetic dynamometer (Cybex Inc, Ronkonkoma, New York, USA). The mechanical and physiological reliability of the Cybex Dynamometer system and testing of the $90^{\circ}$ abducted shoulder position has been previously established. ${ }^{5}$

\section{Isokinetic testing position settings}

Isokinetic testing was performed with the glenohumeral joint in $90^{\circ}$ of abduction in the coronal plane, with a range of motion of $0-90^{\circ}$ of external rotation and $0-65^{\circ}$ of internal rotation. Range of motion stops were used according to the manufacturer's recommendations to ensure that identical ranges of motion were tested bilaterally and during follow up testing. ${ }^{1}$
Subjects were positioned supine on the upper body testing table with stabilisation straps secured at the pelvis and midthoracic levels. An offset handle was provided for the non-tested extremity to grasp during the testing procedure. Gravity correction was not used for the testing position, consistent with the manufacturer's recommendation. ${ }^{15}$ The effect of gravity is almost zero because the value for each half of the two movements is the same but with the opposite effect. The dynamometer input shaft was aligned with the axis of rotation of the glenohumeral joint. The isokinetic test was initiated with the arm in $90^{\circ}$ of external rotation, consistent with the manufacturer's recommendation. ${ }^{15}$

The position of testing used in this study was chosen on the basis of the specificity of muscular function and joint position angles with respect to the throwing motion. ${ }^{16}$ The $90^{\circ}$ abducted position of the glenohumeral joint is a physiological position for this joint and similar to the abduction angle used during the throwing motion. Isokinetic assessment of the internal and external rotators of the shoulder is positional dependent based on the work of Hageman et al, ${ }^{17}$ Soderberg and Blaschak, ${ }^{18}$ and Walmsley and Szybbo. ${ }^{19}$

Changes in muscular strength of the internal and external rotators, based on the length-tension relation of the musculoskeletal system, have been identified objectively in these isokinetic studies and provide a rationale for consistent

Table 2 External rotation peak torque $(\mathrm{Nm})$ for both shoulders at an angular velocity of $60 \%$ sefore and after training

\begin{tabular}{llllll}
\hline & \multicolumn{2}{l}{ Right shoulder } & & \multicolumn{2}{l}{ Left shoulder } \\
\cline { 2 - 3 } & Before & After & & Before & After \\
\hline MJDR group & $23.4(8.5)$ & $26.3(8.5)$ & & $24.5(7.2)$ & $26.8(7.2)$ \\
Isolated group & $24(7.1)$ & $26.4(7.9)$ & & $25.1(7.3)$ & $26.5(7.6)$ \\
Isokinetic group & $21.2(5.5)$ & $24.8(8.2)$ & & $23(6.5)$ & $25.3(8.4)$ \\
Control group & $22.7(7.3)$ & $22.6(7.9)$ & & $23.3(6.4)$ & $23.5(7.6)$ \\
\hline Values are mean (SD). & & & & \\
MJDR, Multijoint dynamic resistance. & & & & \\
\hline
\end{tabular}

Table 3 External rotation peak torque $(\mathrm{Nm})$ for both shoulders at an angular velocity of $120 \% \mathrm{~s}$ before and after training

\begin{tabular}{llllll}
\hline & \multicolumn{2}{l}{ Right shoulder } & & \multicolumn{2}{l}{ Left shoulder } \\
\cline { 2 - 3 } & Before & After & & Before & After \\
\hline MJDR group & $20.6(7.6)$ & $23.5(8.1)$ & & $22(6.9)$ & $24.3(8.1)$ \\
Isolated group & $21.7(6.4)$ & $23.1(6.1)$ & & $21.7(6.3)$ & $23.7(7.0)$ \\
Isokinetic group & $18.5(6.6)$ & $22.1(7.9)$ & & $19.8(5.7)$ & $23.4(8.2)$ \\
Control group & $20.6(7.3)$ & $19.6(7.2)$ & & $19.4(6.7)$ & $20.1(6.1)$ \\
\hline Values are mean (SD). & & & & \\
MJDR, Multijoint dynamic resistance. & & & & \\
\hline
\end{tabular}


Table 4 External rotation peak torque $(\mathrm{Nm})$ for both shoulders at an angular velocity of $180 \%$ s before and after training

\begin{tabular}{llllll}
\hline & \multicolumn{2}{l}{ Right shoulder } & & \multicolumn{2}{l}{ Left shoulder } \\
\cline { 2 - 3 } & Before & After & & Before & After \\
\hline MJDR group & $19.3(7.3)$ & $22.3(9.2)$ & & $19.8(6.7)$ & $21.8(6.6)$ \\
Isolated group & $19.2(6.5)$ & $20.7(6.3)$ & & $18.6(6.1)$ & $20.5(5.8)$ \\
Isokinetic group & $16.3(7.1)$ & $19.4(7.3)$ & & $17.5(7.0)$ & $21(7.3)$ \\
Control group & $19.4(7.9)$ & $16.9(6.6)$ & & $18.3(5.5)$ & $17.2(6.0)$ \\
\hline Values are mean (SD). & & & & \\
MJDR, Multijoint dynamic resistance. & & &
\end{tabular}

application of a standardised test position for isokinetic strength testing. ${ }^{20}$

\section{Velocity settings and data collection}

Testing was performed at angular velocities of 60,120 , and $180 \%$. The same sequence was followed for all the subjects, to increase reliability of data acquisition. ${ }^{9}$ Data used in this study were recorded during three maximal repetitions of internal and external rotation at each angular velocity, ${ }^{21}$ and the best peak torque value at each velocity was used for data analysis.

\section{Isokinetic testing procedure}

Before the test procedure, each subject performed a five minute warm up on an upper body ergometer (Cybex Inc) at an intensity of $600 \mathrm{~kg} / \mathrm{min}$ using the $90 \mathrm{rpm}$ setting. This was followed by passive range of motion of both shoulders in flexion, abduction, $90 \%$ of abduction with external rotation, and $90 \%$ of abduction with internal rotation. The shoulder tested first was randomised to minimise the effects of learning bias. The initial test procedures were also preceded by a period of accommodation to the isokinetic way of exercise (three sets of three repetitions at each angular velocity). Also, three submaximal and one maximal trial repetition at each velocity were performed before each bout to prepare the subject for the test procedure. A 30 second rest was allowed between velocities. Standardised verbal instructions and encouragement were given, with the subjects unable to receive visual feedback during the test procedure. ${ }^{22}$

\section{Experimental design}

The initial isokinetic test procedure was followed by an exercise period of six weeks (three times a week) and followed by the same test procedure. The 48 participants were randomly assigned to one of four groups: three experimental groups, who carried out an exercise strengthening programme, and one control group. All subjects in the experimental groups completed a 10 minute warm up on an upper body ergometer before starting their testing or training protocol.
The first experimental group used the MJDR mode for the internal and external rotators of the shoulder. The MJDR exercise programme consisted of four exercises chosen for strengthening of all the rotator cuff muscle groups: (a) pull ups (or lat pull downs for those who could not execute the pull up); (b) overhead press; (c) reverse pull ups; (d) push ups. Although theses exercises do not involve any rotational activities, they enhance the stabilisation of the glenohumeral joint, which is the main role of the rotator cuff muscles in coordination with the deltoid. ${ }^{23}$ The exercise protocol consisted of 8-15 repetitions in 3-7 sets, with a rest between sets ranging from two to four minutes. The equations of Brzcki $^{24}$ and Mayhew et $a l^{25}$ were used to determine maximal muscular performance at the beginning of the training period and every week thereafter until the exercise period was completed. This assessment determined the number of repetitions and the weight, which changed throughout the exercise period as the subject adapted to the training, in order to obtain submaximal exercise intensity (table 1). The number of sets was the same for all the participants, and they were progressively increased until the end of the exercise period (3-7). The total exercise volume for each participant was computed to be nearly 100 repetitions.

The second experimental group followed a muscle strengthening programme with dumbbells weighing $2 \mathrm{~kg}$ (isolated group). Each subject was positioned as described in the isokinetic mode holding a dumbbell, but this time the exercising shoulder was positioned on a table with stabilisation straps secured at the humeral level obtaining the same axis of rotation as in the isokinetic mode. To train the internal rotation movement, the repetition was initiated with the arm in $90^{\circ}$ of external rotation. Correspondingly, when the external rotation was to be trained, the repetition was initiated with the arm in $90^{\circ}$ of internal rotation. In both cases the trial was completed in the neutral position.

The isokinetic group followed an isokinetic strengthening programme using the isokinetic testing position described above. The isokinetic exercise protocol consisted of 10 repetitions at 90, 120, 180, 210, 240, 240, 210, 180, 120, and $90 \%$. The number of sets and repetitions was chosen

Table 5 Internal rotation peak torque $(\mathrm{Nm})$ for both shoulders at an angular velocity of $60 \%$ before and after training

\begin{tabular}{llllll}
\hline & \multicolumn{2}{l}{ Right shoulder } & & \multicolumn{2}{l}{ Left shoulder } \\
\cline { 2 - 3 } \cline { 5 - 5 } \cline { 5 - 5 } & Before & After & & Before & After \\
\hline MJDR group & $29.5(11.7)$ & $35(11.3)$ & & $27.4(11.1)$ & $30.6(9.5)$ \\
Isolated group & $30.2(10.7)$ & $35(14.0)$ & & $26.8(9.1)$ & $30.2(9.2)$ \\
Isokinetic group & $26.7(9.5)$ & $34.8(10.0)$ & & $24.5(7.5)$ & $32.9(10.5)$ \\
Control group & $27.4(8.3)$ & $30.5(11.5)$ & & $25.4(8.6)$ & $26(9.1)$ \\
\hline Values are mean (SD). & & & \\
MJDR, Multijoint dynamic resistance. & & & \\
\hline
\end{tabular}


Table 6 Internal rotation peak torque $(\mathrm{Nm})$ for both shoulders at an angular velocity of $120 \%$ s before and after training

\begin{tabular}{|c|c|c|c|c|}
\hline & \multicolumn{2}{|c|}{ Right shoulder } & \multicolumn{2}{|l|}{ Left shoulder } \\
\hline & Before & After & Before & After \\
\hline MJDR group & $26.6(10.4)$ & $30.9(10.2)$ & $23.4(10.8)$ & $28.1(10.1)$ \\
\hline Isolated group & $25.5(8.4)$ & 31.5 (11.9) & $20.4(7.7)$ & $25.3(8.4)$ \\
\hline Isokinetic group & $23(8.0)$ & $31.5(9.8)$ & $18.5(6.4)$ & $29.8(10.2)$ \\
\hline Control group & $24.1(7.2)$ & $26.2(10.5)$ & $21.2(8.2)$ & $21.3(8.5)$ \\
\hline
\end{tabular}

according to the total volume accomplished by the other two experimental groups.

The control group had no strength training.

\section{Data analysis}

The peak torque ratios of the external/internal rotators were collected from the isokinetic computer system. One way analysis of variance was used to test the differences in these values between the groups for all angular velocities for the initial tests in both shoulders. Analysis of variance with repeated measures was then used to analyse the peak torque ratios of the external/internal rotators for each shoulder (right and left) before and after the training period separately for each angular velocity.

\section{RESULTS}

One way analysis of variance found no differences in performance between the groups for all angular velocities before the training period. Tables $2-7$ present the absolute mean values for each shoulder and each movement at three angular velocities.

Analysis of variance with repeated measures was used to test the differences in external/internal peak torque ratio before and after training for each group. The independent variable was the group (three experimental groups and one control), and the repeated factors were the test (before and after) and the side (right and left shoulder). Tables 8-10 give the external/internal isokinetic concentric peak torque ratios at three angular velocities for the right and left shoulder.

At $60 \%$, the ratios for the right shoulder were significantly decreased after training for the MJDR and isokinetic groups. For the left shoulder, significantly lower ratios were found for all the experimental groups (table 8). In the isokinetic group, the ratios after training were significantly different from those in the other experimental groups.

At $120 \%$, the strength ratios were similarly lowered in the right shoulder after training, meaning that there were no significant differences in restoration of the ratios between the groups (table 9). For the left shoulder, the experimental groups showed significant restoration of the ratios after training, and the difference between groups was significant.
Specifically, the decrease in ratio in the isokinetic group was significantly different from that in the isolated group.

At $180 \%$ angular velocity, all experimental groups had significantly lower ratios in both limbs, and there were significant differences between the groups for both limbs. Again, the isokinetic group showed a significantly greater decrease in the ratios than the other experimental groups (table 10).

\section{DISCUSSION}

Altering the strength of the external/internal rotators in order to restore muscle imbalances was the main accomplishment of this study. In both shoulders, the external/internal rotator ratio was higher than normal $(112 \% v 81 \%)$ in the initial test, indicating that the strengthening programme needed to focus on the internal rotators. The values after the test varied from $70 \%$ to $94 \%$, indicating that all the training types were effective in altering the strength ratios. The isokinetic training seemed to produce significantly greater changes in strength ratio than the other types, indicating that it was the most effective (tables 8-10). However, a major limitation of this study is that the greater improvement shown by the isokinetic group may be due to the similarity of the movement in the training protocol to that in the evaluation test. However, this is the most reliable and standard method for assessing the two muscle groups at the same time in order to detect muscle imbalances.

These results are in partial agreement with previous investigations identifying greater improvements in muscle performance after isokinetic strengthening than after isotonic exercise programmes. ${ }^{12} 26$ In particular, Smith and Melton ${ }^{26}$ found isokinetic exercise to be more advantageous than isotonic training for quadriceps and hamstrings. In 12 adolescent boys, all groups improved in strength measured isokinetically, and the high speed isokinetic group showed the greatest improvements in all functional tests.

Beneka et $a l^{4}$ compared the effectiveness of isokinetic exercise and MJDR training in increasing peak torque separately for the external and internal rotators of the shoulder. They concluded that both methods resulted in significant strength improvement, although the isokinetic method was more effective at increasing internal rotator

Table 7 Internal rotation peak torque $(\mathrm{Nm})$ for both shoulders at an angular velocity of $180 \%$ s before and after training

\begin{tabular}{llllll}
\hline & \multicolumn{2}{l}{ Right shoulder } & & \multicolumn{2}{l}{ Left shoulder } \\
\cline { 2 - 3 } \cline { 5 - 6 } & Before & After & & Before & After \\
\hline MJDR group & $23.7(10.0)$ & $27.9(10.3)$ & & $21(10.0)$ & $25.6(9.3)$ \\
Isolated group & $24.2(7.9)$ & $27.2(9.9)$ & & $18.2(6.9)$ & $21.7(8.2)$ \\
Isokinetic group & $19.7(8.7)$ & $29(9.7)$ & & $16.2(7.2)$ & $27.9(9.0)$ \\
Control group & $21.7(8.3)$ & $23.2(9.9)$ & & $18.5(6.9)$ & $18.9(7.5)$ \\
\hline
\end{tabular}

Values are mean (SD).

MJDR, Multijoint dynamic resistance. 
Table 8 External/internal peak torque ratios for both shoulders at an angular velocity of $60 \% \mathrm{~s}$ before and after training

\begin{tabular}{|c|c|c|c|c|}
\hline & \multicolumn{2}{|c|}{ Right shoulder } & \multicolumn{2}{|l|}{ Left shoulder } \\
\hline & Before & After & Before & After \\
\hline $\begin{array}{l}\text { MJDR group } \\
\text { Isolated group } \\
\text { Isokinetic group } \\
\text { Control group }\end{array}$ & $\begin{array}{l}81.1(13) \\
81.3(14.1) \\
82.3(16.1) \\
82.5(8.7)\end{array}$ & $\begin{array}{l}75.6(10.8)^{*} \\
78.6(13.2) \\
71.7(8.7)^{*} \\
83.2(8.8)\end{array}$ & $\begin{array}{l}94.2(19.2) \\
96.6(19.6) \\
94.8(17.2) \\
87.8(9.1)\end{array}$ & $\begin{array}{l}89.2(10.8)^{*} \\
89.0(14.7)^{*} \\
78.0(10.0)^{*} \dagger \S \\
89.8(6.9)\end{array}$ \\
\hline
\end{tabular}

Values are mean (SD).

*Significantly different from before training $(p<0.05)$.

$\dagger$ Significantly different from the MJDR group $(p<0.05)$.

†Significantly different from the isokinetic group $(p<0.05)$.

$\S$ Significantly different from the isolated group $(p<0.05)$

Significantly different from the control group $(p<0.05)$

MJDR, Multijoint dynamic resistance.

strength. It is these results that agree with the results of the present study. The restoration of imbalances may be due to strengthening of both muscle groups, but especially the internal rotators.

One can argue that adaptations caused by different training regimens (dynamic resistance, free weights, or isokinetic) are not directly comparable because the total work performed is not exactly the same in the three groups and this is an important limitation of the study. Furthermore, it should be noted that it is difficult to compare strength gains among various strength training modes. The initial performance levels, the volume and intensity of the exercise, and the equipment used (isokinetic or resistance machines versus free weights) all affect outcomes and cannot be completely controlled. For that reason, we chose to begin the experimental phase of the study with the MJDR and the isolated group in order to estimate the total exercise volume that the participants had to accomplish to obtain submaximal intensity.

In this study, the intensity of exercise in the isokinetic group was submaximal, and in the other two experimental groups submaximal intensity was determined using the equations of Brzcki $^{24}$ and Mayhew. ${ }^{25}$ It has also been suggested that it is not the intensity of the contraction alone, ${ }^{27}$ but the total load (table 1 ) or the practising of a certain movement that increases maximal strength. ${ }^{28}{ }^{29}$ Thus, less discomfort resulting from submaximal intensity and the reduced effort involved make isolated exercise or MJDR training a viable alternative for sedentary people who want to increase shoulder rotator strength and restore possible imbalances.

Comparing the performance of the two dynamic resistance groups, in the isolated group, the strength ratio was significantly changed only for the left shoulder, which had

Table 9 External/internal peak torque ratios for both shoulders at an angular velocity of $120 \%$ s before and after training

\begin{tabular}{|c|c|c|c|c|}
\hline & \multicolumn{2}{|c|}{ Right shoulder } & \multicolumn{2}{|l|}{ Left shoulder } \\
\hline & Before & After & Before & After \\
\hline MJDR group & $78.8(13.2)$ & $76.4(8.5)^{*}$ & $101.4(25.6)$ & $87.4(16.1)^{*}$ \\
\hline Isolated group & $87.1(16.5)$ & $77.1(16.2)^{*}$ & $112.1(23.7)$ & $94.2(16.3)^{*}$ \\
\hline Isokinetic group & $80.4(15.6)$ & $69(7.5)^{*}$ & 107.9 (18.3) & $78.9(12.1)^{*} \S \oplus$ \\
\hline Control group & $84(14.1)$ & $82.7(17.6)$ & $94.2(16.02)$ & $96.2(16.3)$ \\
\hline
\end{tabular}

Values are mean (SD)

* Significantly different from before training $(p<0.05)$

†Significantly different from the MJDR group $(p<0.05)$.

$¥$ Significantly different from the isokinetic group $(p<0.05)$.

$\S$ Significantly different from the isolated group $(p<0.05)$.

- Significantly different from the control group $(p<0.05)$.

MJDR, Multijoint dynamic resistance.

Table 10 External/internal peak torque ratios for both shoulders at an angular velocity of $180 \%$ s before and after training

\begin{tabular}{|c|c|c|c|c|}
\hline & \multicolumn{2}{|c|}{ Right shoulder } & \multicolumn{2}{|l|}{ Left shoulder } \\
\hline & Before & After & Before & After \\
\hline $\begin{array}{l}\text { MJDR group } \\
\text { Isolated group } \\
\text { Isokinetic group } \\
\text { Control group }\end{array}$ & $\begin{array}{l}86.8(12.1) \\
81.9(14.0) \\
81.4(23.3) \\
80.9(19.8)\end{array}$ & $\begin{array}{l}79.04(16.3)^{*} \\
69.04(9.9)^{*} \\
65.6(6.7)^{\star} \S \\
82.9(21.5)\end{array}$ & $\begin{array}{l}103.4(29.3) \\
102.8(17.3) \\
110.1(28.4) \\
100.5(19.4)\end{array}$ & $\begin{array}{l}85.1(19.9)^{*} \\
81.4(13.3)^{*} \\
75.1(8.8)^{*} \S \\
100.3(19.6)\end{array}$ \\
\hline
\end{tabular}

Values are mean (SD)

*Significantly different from before training $(p<0.05)$.

†Significantly different from the MJDR group $(p<0.05)$.

$\ddagger$ Significantly different from the isokinetic group $(p<0.05)$.

$\S$ Significantly different from the isolated group $(p<0.05)$.

- Significantly different from the control group $(p<0.05)$.

MJDR, Multijoint dynamic resistance. 
weaker internal rotators before training. In contrast, in the MJDR group the ratios were altered significantly for both shoulders (table 8 ). These conclusions are in agreement with the study of Blievernicht, ${ }^{30}$ who stated that muscle groups that fire in an uncoordinated fashion can primarily be restored using isolated movements. He reported that muscles that are strong and short are lengthened and inhibited using active range of motion. Typically, actively shortening a passive muscle will lengthen and inhibit its dominant antagonist. Thus, according to Blievernicht, a single exercise can be used to stretch one muscle and strengthen another. He also stated that, as muscle balance improves, more complex movements should replace isolated exercises.

The last statement seems to agree with our results showing significant improvement in both sides in the group that exercised with closed kinetic chain exercise. Although there was an improvement in muscle balance when the isolated exercise programme was applied, further strengthening modes were needed to obtain more impressive increases in performance. Blievernicht $t^{30}$ proposed that, after the initial strength improvement is assessed, isolated exercises should be replaced with more complex movements in order to obtain more complete performance-that is, the same conclusion as this study.

Furthermore, Kibler ${ }^{31}$ reported that closed chain techniques can increase the effectiveness of rehabilitation protocols because they allow more normal physiological activation and biomechanical motions, especially in the early rehabilitation phase. They have been shown to be effective in knee/leg rehabilitation, but are also useful in shoulder/scapula rehabilitation.

More recent studies ${ }^{32}$ also used closed kinetic chain exercise protocols extensively in rehabilitation of shoulder injuries. They are felt to be preferable to other exercise programmes because they simulate physiological and biomechanical functions, create little shear stress across injured or healing joints, and reproduce proprioceptive stimuli. Because of these advantages, they may be used early in rehabilitation and have been integral parts of "accelerated" rehabilitation programmes. ${ }^{32}$

Our results confirm the findings of Campbell and Glenn,,33 who reported a significant increase in quadriceps muscle strength as well as hamstring muscle strength as a result of a seven week open kinetic chain rehabilitation programme. Also Witvrouw ${ }^{34}$ found significant strength increases in the quadriceps and hamstrings muscles in two rehabilitation groups (exercising with open or closed kinetic chain exercise) suffering from patellofemoral pain.

Observing the performance in both limbs, the bilateral difference is obvious before and after training and at all angular velocities. Comparing the mean values for the two limbs (tables 2-7), we concluded that the external rotators of the left shoulder are stronger than those of the right. This difference is logical as many studies report that the external rotators of the non-dominant side are stronger than those of the dominant side in both collegiate and professional level athletes. ${ }^{75}$ Comparing the mean ratio for the three angular velocities tested, it seems that, in both limbs, at greater velocity the ratios are almost the same or slightly increased. This result is in accordance with previous studies comparing external/internal rotator ratios in the same testing position. It has been reported that the ratios rise as the angular velocity increases. More precisely, Alderink and $\mathrm{Kuck}^{36}$ tested pitchers and found that the biggest ratio was calculated for $300 \%$ angular velocity and the smallest ratio for $90 \%$. Cook et $\mathrm{al}^{37}$ and Hinton ${ }^{38}$ came to the same conclusion after testing pitchers and non-pitchers. In contrast, Ivey et $a l^{39}$ found that the ratios decreased with faster velocities in exercisers and non-exercisers.
What is already known on this topic

The need to detect muscle imbalances and alter strength ratios in the shoulder joint is well established. Many studies have examined the effectiveness of different training modes for improving the performance of muscles, including the rotator cuff muscles.

\section{What this study adds}

This study compares the effectiveness of different training modes in altering shoulder strength ratios and gives athletes who use that particular joint to perform athletic moves-for example, pitchers and tennis players - valuable information about how to apply these protocols to be more efficient and minimise the risk of injury.

\section{CONCLUSION}

We suggest that trainers of athletes who particularly use the shoulder to perform their sport-for example, pitchers, tennis players - can help them be more efficient and minimise the risk of injury by applying simple strength training protocols. All the exercise types studied were effective in altering the strength ratios, but isokinetic training appeared to be significantly more effective. If isokinetic equipment is not available, isolated or MJDR training is a practical alternative for athletes who want to increase shoulder rotator strength and restore any muscle imbalances.

\section{Authors' affiliations}

P C Malliou, K Giannakopoulos, A G Beneka, A Gioftsidou,

G Godolias, Department of Physical Education and Sport Science, Democritus University of Thrace, Komotini, Greece

Conflict of interest: none declared

\section{REFERENCES}

1 Fleisig GS, Andrews JR, Dillman CJ, et al. Kinetics of baseball pitching with implications about injury mechanisms. Am J Sports Med 1995;23:233-9.

2 Sahrmann SA. Posture and muscle imbalance. Faulty lumbar pelvic alignments. Phys Ther 1987;67:1840-4.

3 Caillet R. Soft tissue pain and disability. Philadelphia: FA Davis, 1977.

4 Beneka A, Malliou P, Giannakopoulos K, et al. Different training modes for the rotator cuff muscle group. A comparative study. Isokinet Exerc Sci 2002;10:73-9

5 Brostrom LA, Kronberg M, Nemeth G, et al. The effect of shoulder muscle training in patients with recurrent shoulder dislocations. Scand J Rehabil Med 1992;24:11-15.

6 Ellenbecker TS, Mattalino A. Concentric isokinetic shoulder internal and external rotation strength in professional baseball pitchers. J Orthop Sports Phys Ther 1997;25:323-8.

7 Meister K. Injuries to the shoulder in the throwing athlete. Am J Sports Med 2000;28:265-75

8 Sirota SC, Malanga GA, Eischen JJ. An eccentric and concentric strength profile of shoulder external and internal rotator muscles in professional baseball pitchers. Am J Sports Med 1997;25:59-64.

9 Wilhite MR, Cohen ER, Wilhite SC. Reliability of concentric and eccentric measurements of quadriceps performance using the Kim-Com dynamometer: the effects of testing order for three different speeds. J Orthop Sports Phys Ther 1992;15:175-82.

10 Kibler WB, Chandler TJ, Livingston BP, et al. Shoulder range of motion in elite tennis players. Effect of age and years of tournament play. Am J Sports Med 1996;24:279-85

11 Mikeksy AE, Edwards JE, Wigglesworth JK. Eccentric and concentric strength of the shoulder and arm musculature in collegiate baseball pitchers. Am J Sports Med 1995;23:638-42.

12 Greenleaf JE, Bernauer EM, Ertl AC, et al. Work capacity during 30 days' of bed rest with isotonic and isokinetic exercise training. J Appl Physiol 1989;67:1820.

13 Hakkinen K, Kauhanen P. Scientific evaluation of specific loading of the knee extensors with variable resistance, isokinetic and barbell exercises. In: Marconnet $P$, Komi PV, eds. Muscular function in exercise and training. Basel: Karger S, 1987:224. 
14 Vandervoort AA Sale DG, Moroz GR. Strength-velocity relationship and fatigability of unilateral versus bilateral arm extension. Eur J Appl Phys 1987;56:201-5.

15 Cybex Inc. Cybex Applications Manual 300 Series Dynamometer System. Ronkonkoma, NY: Cybex Inc, 1991.

16 Bassett RW, Browne AO, Morrey BF, et al. Glenohumeral muscle force and moment mechanics in a position of shoulder instability. J Biomech 1990;23:405-15.

17 Hageman PA, Mason DK, Rydlund KW, et al. Effects of position and speed on eccentric and concentric isokinetic testing of the shoulder rotators. J Orthop Sports Phys Ther 1989;1 1:64-9.

18 Soderberg GJ, Blaschak MJ. Shoulder internal and external rotation peak torque production through a velocity spectrum in differing positions. J Orthop Sports Phys Ther 1987:8:518-24.

19 Walmsley RP, Szybbo C. A comparative study of the torque generated by the shoulder internal and external rotator muscles in different positions and at varying speeds. J Orthop Sports Phys Ther 1987;9:217-22.

20 Ellenbecker TS, Davies GJ, Rowinski MJ. Concentric versus eccentric isokinetic strengthening of the rotator cuff: objective data versus functional test. Am J Sports Med 1988;16:64-9.

21 Wilk KE, Arrigo CA. Peak torque and maximum work repetition during isokinetic testing of the shoulder internal and external rotators. Isokinet Exerc Sci 1994;4:171-5.

22 Baltzopoulos V, Williams JG, Brodie DA. Sources of error in isokinetic dynamometry: effects of visual feedback on maximum torque measurements. J Orthop Sports Phys Ther 1991;13:138-42.

23 Wilk K. Conditioning and training techniques. In: Hawkins RJ, Misamore GW eds. Shoulder injuries in the athlete. Edinburgh: Churchill-Livingstone, 1996:340.

24 Brzycki M. Strength testing: predicting a one rep max from reps-to-fatigue. Journal of Physical Education, Recreation and Dance 1993 Jan:88-90.

25 Mayhew J, Ware J, Prinster J. Using lift repetitions to predict muscular strength in adolescent males. National Strength and Conditioning Association Journal 1993:15:35-38.
26 Smith MJ, Melton P. Isokinetic versus isotonic variable-resistance training. Am J Sports Med 1981;9:275.

27 McDonagh MJN, Davies CTM. Adaptive response of mammalian skeletal muscle to exercise with high loads. Eur J Appl Physiol 1984:52:139-55.

28 Almasbakk B, Hoff J. Coordination, the determinant of velocity specificity? J Appl Physiol 1996;86:2046-52

29 Hortobagyi T, Tunnel D, Moody J, et al. Low- or high-intensity strength training partially restores impaired quadriceps force accuracy and steadiness in aged adults. J Gerontol 2001;56A:B38-47.

30 Blievernicht JA. Round shoulder syndrome. IDEA health and fitness source Sept 2000.

31 Kibler WB. Closed kinetic chain rehabilitation for sports injuries. Phys Med Rehabil Clin N Am 2000;1 1:369-84.

32 Kibler WB, Livingston B. Closed chain rehabilitation for upper and lower extremities. J Am Acad Orthop Surg 2001 ;:412-21.

33 Campbell DE, Glenn W. Rehabilitation of knee flexor and knee extensor muscle strength in patients with meniscectomies, ligamentous repairs, and chondromalacia. Phys Ther 1982;62:10-15.

34 Witvrouw E, Lysens R, Bellemans J, et al. Open versus closed kinetic chain exercises for patellofemoral pain. Am J Sports Med 2000;28:687-94.

35 Wilk KE, Andrews JR, Arrigo CA. The abductor and adductor strength characteristics of professional baseball pitchers. Am J Sports Med 1995;23:307-11.

36 Alderink GJ, Kuck DJ. Isokinetic shoulder strength of high school and collegeaged pitchers. J Orthop Sports Phys Ther 1986;7:163-72.

37 Cook EE, Gray VL, Savinar-Nogue E, et al. Shoulder antagonistic strength ratios: a comparison between college-level baseball pitchers and nonpitchers. J Orthop Sports Phys Ther 1987:8:451-61.

38 Hinton RY. Isokinetic evaluation of shoulder rotational strength in high school baseball pitchers. Am J Sports Med 1988;16:274-9.

39 Ivey FM, Calhoun JH, Rusche K, et al. Isokinetic testing of shoulder strength normal values. Arch Phys Med Rehabil 1985;66:384-6.

\section{Call for papers}

\section{Themed issue on Sports Injury Prevention}

British Journal of Sports Medicine will publish a special issue on sports injury prevention in June 2005 to coincide with the $1^{\text {st }}$ World Congress on Sports Injury Prevention (Oslo, Norway, 23-25 June; http://www.ostrc.no/congress2005/).

We welcome original submissions for this themed issue - all articles should be submitted via http://bjsm.bmijournals.com/ (our electronic submission and review system) no later than $1^{\text {st }}$ January 2005. Please indicate in your covering letter that you wish to be considered for the special issue (all articles will be subject to the normal peer review process).

\section{Congress abstracts}

We are delighted to announce that the abstracts for the $1^{\text {st }}$ World Congress on Sports Injury Prevention will be published in this special issue of British Journal of Sports Medicine; the journal will be available to all congress delegates in Oslo. Abstracts should be submitted on the congress website before $1^{\text {st }}$ February 2005.

Please note that BJSM will be published monthly from January 2005 